\title{
Classes of operators satisfying $a$-Weyl's theorem
}

\author{
by \\ Pietro Aiena (Palermo)
}

\begin{abstract}
In this article Weyl's theorem and $a$-Weyl's theorem on Banach spaces are related to an important property which has a leading role in local spectral theory: the single-valued extension theory.

We show that if $T$ has SVEP then Weyl's theorem and $a$-Weyl's theorem for $T^{*}$ are equivalent, and analogously, if $T^{*}$ has SVEP then Weyl's theorem and $a$-Weyl's theorem for $T$ are equivalent. From this result we deduce that $a$-Weyl's theorem holds for classes of operators for which the quasi-nilpotent part $H_{0}(\lambda I-T)$ is equal to $\operatorname{ker}(\lambda I-T)^{p}$ for some $p \in \mathbb{N}$ and every $\lambda \in \mathbb{C}$, and for algebraically paranormal operators on Hilbert spaces. We also improve recent results established by Curto and Han, Han and Lee, and Oudghiri.
\end{abstract}

1. Notation and terminology. We begin with some standard notations in Fredholm theory. Throughout this note by $L(X)$ we denote the algebra of all bounded linear operators acting on an infinite-dimensional complex Banach space $X$. For every $T \in L(X)$ we denote by $\alpha(T)$ and $\beta(T)$ the dimension of the kernel ker $T$ and the codimension of the range $T(X)$, respectively. The class of upper semi-Fredholm operators is defined by

$$
\Phi_{+}(X):=\{T \in L(X): \alpha(T)<\infty \text { and } T(X) \text { is closed }\},
$$

whilst the class of lower semi-Fredholm operators is defined by

$$
\Phi_{-}(X):=\{T \in L(X): \beta(T)<\infty\} .
$$

An operator $T \in L(X)$ is said to be semi-Fredholm if $T \in \Phi_{+}(X) \cup \Phi_{-}(X)$, whilst the class of Fredholm operators is $\Phi(X):=\Phi_{+}(X) \cap \Phi_{-}(X)$. The index of a semi-Fredholm operator is defined by ind $T:=\alpha(T)-\beta(T)$.

For a linear operator $T$ the ascent $p:=p(T)$ is defined as the smallest nonnegative integer $p$ such that $\operatorname{ker} T^{p}=\operatorname{ker} T^{p+1}$. If such an integer does not exist we put $p(T)=\infty$. Analogously, the descent $q:=q(T)$ is defined as the smallest nonnegative integer $q$ such that $T^{q}(X)=T^{q+1}(X)$, and if such an integer does not exist we put $q(T)=\infty$. A classical result states that if

2000 Mathematics Subject Classification: Primary 47A10, 47A11; Secondary 47A53, 47A55.

Key words and phrases: single-valued extension property, Fredholm theory, Weyl's theorems, algebraically paranormal operators. 
$p(T)$ and $q(T)$ are both finite then $p(T)=q(T)$ (see [24, Proposition 38.3]). Moreover, $\lambda \in \sigma(T)$ (the spectrum of $T$ ) is a pole of the resolvent precisely when $0<p(\lambda I-T)=q(\lambda I-T)<\infty$ (see Proposition 50.2 of [24]), and in this case $X=\operatorname{ker}(\lambda I-T)^{p} \oplus(\lambda I-T)^{p}(X)$, with $p:=p(\lambda I-T)=q(\lambda I-T)$. Two important classes of operators in Fredholm theory are the class of upper semi-Browder operators defined by

$$
\mathcal{B}_{+}(X):=\left\{T \in \Phi_{+}(X): p(T)<\infty\right\},
$$

and the class of lower semi-Browder operators defined by

$$
\mathcal{B}_{-}(X):=\left\{T \in \Phi_{-}(X): q(T)<\infty\right\}
$$

The class of Browder operators (known in the literature also as the RieszSchauder operators) is defined by $\mathcal{B}(X):=\mathcal{B}_{+}(X) \cap \mathcal{B}_{-}(X)$. A bounded operator $T \in L(X)$ is called a Weyl operator if $T \in \Phi(X)$ and ind $T=0$. A Browder operator $T$ is Weyl since the finiteness of $p(T)$ and $q(T)$ entails for a Fredholm operator $T$ that $T$ has index 0 (cf. [24, Proposition 38.5]).

The classes of operators defined above motivate the definition of several spectra. The upper semi-Browder spectrum of $T \in L(X)$ is defined by

$$
\sigma_{\mathrm{ub}}(T):=\left\{\lambda \in \mathbb{C}: \lambda I-T \notin \mathcal{B}_{+}(X)\right\},
$$

the lower semi-Browder spectrum of $T \in L(X)$ is defined by

$$
\sigma_{\mathrm{lb}}(T):=\left\{\lambda \in \mathbb{C}: \lambda I-T \notin \mathcal{B}_{-}(X)\right\},
$$

whilst the Browder spectrum of $T \in L(X)$ is defined by

$$
\sigma_{\mathrm{b}}(T):=\{\lambda \in \mathbb{C}: \lambda I-T \notin \mathcal{B}(X)\} .
$$

Finally, the Weyl spectrum of $T \in L(X)$ is defined by

$$
\sigma_{\mathrm{w}}(T):=\{\lambda \in \mathbb{C}: \lambda I-T \text { is not Weyl }\} .
$$

It should be noted that $\sigma_{\mathrm{w}}(T)=\sigma_{\mathrm{w}}\left(T^{*}\right)$, whilst

$$
\sigma_{\mathrm{ub}}(T)=\sigma_{\mathrm{lb}}\left(T^{*}\right), \quad \sigma_{\mathrm{lb}}(T)=\sigma_{\mathrm{ub}}\left(T^{*}\right) .
$$

Moreover,

$$
\sigma_{\mathrm{w}}(T) \subseteq \sigma_{\mathrm{b}}(T)=\sigma_{\mathrm{w}}(T) \cup \operatorname{acc} \sigma(T),
$$

where we write acc $K$ for the accumulation points of $K \subseteq \mathbb{C}$.

Recall that $T \in L(X)$ is said to be bounded below if $T$ is injective and has closed range. Let $\sigma_{\mathrm{a}}(T)$ denote the classical approximate point spectrum of $T$ defined as

$$
\sigma_{\mathrm{a}}(T):=\{\lambda \in \mathbb{C}: \lambda I-T \text { is not bounded below }\},
$$

and let

$$
\sigma_{\mathrm{S}}(T):=\{\lambda \in \mathbb{C}: \lambda I-T \text { is not surjective }\}
$$

denote the surjectivity spectrum of $T$. 
For a bounded operator $T \in L(X)$ set

$$
p_{00}(T):=\sigma(T) \backslash \sigma_{\mathrm{b}}(T)=\{\lambda \in \sigma(T): \lambda I-T \text { is Browder }\} .
$$

and, if we write iso $K$ for the set of all isolated points of $K \subseteq \mathbb{C}$, then we define

$$
\pi_{00}(T):=\{\lambda \in \text { iso } \sigma(T): 0<\alpha(\lambda I-T)<\infty\} .
$$

Obviously,

$$
p_{00}(T) \subseteq \pi_{00}(T) \quad \text { for every } T \in L(X) .
$$

Following Coburn [10], we say that Weyl's theorem holds for $T \in L(X)$ if

$$
\sigma(T) \backslash \sigma_{\mathrm{w}}(T)=\pi_{00}(T),
$$

whilst $T$ satisfies Browder's theorem if

$$
\sigma(T) \backslash \sigma_{\mathrm{w}}(T)=p_{00}(T),
$$

or equivalently, $\sigma_{\mathrm{w}}(T)=\sigma_{\mathrm{b}}(T)$.

The Weyl (or essential) approximate point spectrum $\sigma_{\mathrm{wa}}(T)$ of a bounded operator $T \in L(X)$ is the complement of those $\lambda \in \mathbb{C}$ for which $\lambda I-T \in$ $\Phi_{+}(X)$ and $\operatorname{ind}(\lambda I-T) \leq 0$. Note that $\sigma_{\mathrm{wa}}(T)$ is the intersection of all approximate point spectra $\sigma_{\mathrm{a}}(T+K)$ of compact perturbations $K$ of $T$ (see [32]). The Weyl surjectivity spectrum $\sigma_{\mathrm{ws}}(T)$ is the complement of those $\lambda \in \mathbb{C}$ for which $\lambda I-T \in \Phi_{-}(X)$ and $\operatorname{ind}(\lambda I-T) \geq 0$. The spectrum $\sigma_{\text {wa }}(T)$ coincides with the intersection of all surjectivity spectra $\sigma_{\mathrm{s}}(T+K)$ of compact perturbations $K$ of $T$ (see [32] or [1, p. 151]). Clearly, the two spectra are dual to each other, i.e.,

$$
\sigma_{\mathrm{wa}}(T)=\sigma_{\mathrm{ws}}\left(T^{*}\right) \quad \text { and } \quad \sigma_{\mathrm{ws}}(T)=\sigma_{\mathrm{wa}}\left(T^{*}\right) .
$$

Furthermore, $\sigma_{\mathrm{w}}(T)=\sigma_{\mathrm{wa}}(T) \cup \sigma_{\mathrm{ws}}(T)$. Note that $\sigma_{\mathrm{wa}}(T) \subseteq \sigma_{\mathrm{ub}}(T)$ and $\sigma_{\mathrm{ws}}(T) \subseteq \sigma_{\mathrm{lb}}(T)$; precisely:

$$
\begin{aligned}
\sigma_{\mathrm{ub}}(T) & =\sigma_{\mathrm{wa}}(T) \cup \operatorname{acc} \sigma_{\mathrm{a}}(T), \\
\sigma_{\mathrm{lb}}(T) & =\sigma_{\mathrm{ws}}(T) \cup \operatorname{acc} \sigma_{\mathrm{s}}(T)
\end{aligned}
$$

(see [33]). Define

$$
\pi_{00}^{\mathrm{a}}(T):=\left\{\lambda \in \text { iso } \sigma_{\mathrm{a}}(T): 0<\alpha(\lambda I-T)<\infty\right\} .
$$

Following Rakočević [32], we shall say that $a$-Weyl's theorem holds for $T \in$ $L(X)$ if

$$
\sigma_{\mathrm{a}}(T) \backslash \sigma_{\mathrm{wa}}(T)=\pi_{00}^{\mathrm{a}}(T),
$$

whilst we shall say that $T$ satisfies $a$-Browder's theorem if

$$
\sigma_{\mathrm{wa}}(T)=\sigma_{\mathrm{ub}}(T) .
$$

We have

$$
a \text {-Browder's theorem } \Rightarrow \text { Browder's theorem, }
$$


and

$a$-Weyl's theorem $\Rightarrow$ Weyl's theorem $\Rightarrow$ Browder's theorem (see for instance [1, Chapter 3]).

2. Single-valued extension property. The single-valued extension property dates back to the early days of local spectral theory and was introduced by Dunford [19], in his theory of spectral operators. This property plays a crucial role in local spectral theory (see the recent monograph of Laursen and Neumann [26]). We shall consider a local version of this property, which has been studied in recent papers [3], [4], [6], and previously by Finch [20] and Mbekhta [29].

Definition 2.1. The operator $T \in L(X)$ is said to have the singlevalued extension property at $\lambda_{0} \in \mathbb{C}$ (abbreviated SVEP at $\lambda_{0}$ ) if for every open disc $U$ centered at $\lambda_{0}$ the only analytic function $f: U \rightarrow X$ which satisfies the equation $(\lambda I-T) f(\lambda)=0$ for all $\lambda \in U$ is the function $f \equiv 0$.

An operator $T \in L(X)$ is said to have SVEP if $T$ has SVEP at every point $\lambda \in \mathbb{C}$.

Trivially, an operator $T \in L(X)$ has SVEP at every point of the resolvent set $\varrho(T):=\mathbb{C} \backslash \sigma(T)$. Moreover, from the identity theorem for analytic functions it easily follows that $T \in L(X)$ has SVEP at every point of the boundary $\partial \sigma(T)$ of the spectrum $\sigma(T)$. In particular, every operator has SVEP at the isolated point of its spectrum.

An important subspace in local spectral theory is the glocal spectral subspace $\mathcal{X}_{T}(F)$ associated with a closed subset $F \subseteq \mathbb{C}$. It is defined, for an arbitrary operator $T \in L(X)$ and a closed subset $F$ of $\mathbb{C}$, as the set of all $x \in X$ for which there exists an analytic function $f: \mathbb{C} \backslash F \rightarrow X$ which satisfies the identity $(\lambda I-T) f(\lambda)=x$ for all $\lambda \in \mathbb{C} \backslash F$. The basic role of SVEP arises in local spectral theory since all decomposable operators enjoy this property. Recall $T \in L(X)$ has the decomposition property $(\delta)$ if $X=\mathcal{X}_{T}(\bar{U})+\mathcal{X}_{T}(\bar{V})$ for every open cover $\{U, V\}$ of $\mathbb{C}$. Decomposable operators may be defined in several ways, for instance as the union of those with property $(\beta)$ and property $(\delta)$ (see [26, Theorem 2.5.19] for relevant definitions). Note that property $(\beta)$ implies that $T$ has SVEP, whilst property $(\delta)$ implies SVEP for $T^{*}$ (see [26, Theorem 2.5.19]).

Note that

$$
p(\lambda I-T)<\infty \Rightarrow T \text { has SVEP at } \lambda
$$

and dually

$$
q(\lambda I-T)<\infty \Rightarrow T^{*} \text { has SVEP at } \lambda
$$

(see [5]). Furthermore,

$$
\sigma_{\mathrm{a}}(T) \text { does not cluster at } \lambda \Rightarrow T \text { has SVEP at } \lambda,
$$


and

$$
\sigma_{\mathrm{s}}(T) \text { does not cluster at } \lambda \Rightarrow T^{*} \text { has SVEP at } \lambda
$$

(see $[6])$.

Let us consider the quasi-nilpotent part of $T$, i.e. the set

$$
H_{0}(T):=\left\{x \in X: \lim _{n \rightarrow \infty}\left\|T^{n} x\right\|^{1 / n}=0\right\} .
$$

It is easily seen that $\operatorname{ker}\left(T^{m}\right) \subseteq H_{0}(T)$ for every $m \in \mathbb{N}$ and $T$ is quasinilpotent if and only if $H_{0}(T)=X$ (see [29, Remarque 1.1]). Moreover, if $T$ is invertible then $H_{0}(T)=\{0\}$.

The analytic core of $T$ is the set $K(T)$ of all $x \in X$ such that there exists a sequence $\left(u_{n}\right) \subset X$ and $\delta>0$ for which $x=u_{0}$, and $T u_{n+1}=u_{n}$ and $\left\|u_{n}\right\| \leq \delta^{n}\|x\|$ for every $n \in \mathbb{N}$. It easily follows, from the definition, that $K(T)$ is a linear subspace of $X$ and $T(K(T))=K(T)$.

Definition 2.2. An operator $T \in L(X), X$ a Banach space, is said to be semi-regular if $T(X)$ is closed and $\operatorname{ker} T \subseteq T^{\infty}(X)$. An operator $T \in L(X)$ is said to admit a generalized Kato decomposition, abbreviated GKD, if there exists a pair $(M, N)$ of $T$-invariant closed subspaces such that $X=M \oplus N$, the restriction $T \mid M$ is semi-regular and $T \mid N$ is quasi-nilpotent.

A relevant case is obtained if we assume in the definition above that $T \mid N$ is nilpotent. In this case $T$ is said to be of Kato type (see for details [1]). Recall that every semi-Fredholm operator is of Kato type, by the classical result of Kato [25] (see also Chapter 1 of [1]). The following characterizations of SVEP for operators of Kato type have been proved in [3] and [6] (see also Chapter 3 in [1]).

TheOREM 2.3. If $\lambda_{0} I-T \in L(X)$ is of Kato type then the following statements are equivalent:

(i) Thas SVEP at $\lambda_{0}$;

(ii) $p\left(\lambda_{0} I-T\right)<\infty$;

(iii) $\sigma_{\mathrm{a}}(T)$ does not cluster at $\lambda_{0}$;

(iv) $H_{0}\left(\lambda_{0} I-T\right)$ is closed.

If $\lambda_{0} I-T$ is semi-Fredholm then assertions (i)-(iv) are equivalent to the following statement:

(v) $H_{0}\left(\lambda_{0} I-T\right)$ is finite-dimensional.

Dually, if $\lambda_{0} I-T$ is of Kato type then the following statements are equivalent:

(vi) $T^{*}$ has SVEP at $\lambda_{0}$;

(vii) $q\left(\lambda_{0} I-T\right)<\infty$;

(viii) $\sigma_{\mathrm{s}}(T)$ does not cluster at $\lambda_{0}$; 
If $\lambda_{0} I-T$ is semi-Fredholm then assertions (vi)-(viii) are equivalent to the following statement:

(ix) $K\left(\lambda_{0} I-T\right)$ is finite-codimensional.

Let $\lambda_{0}$ be an isolated point of $\sigma(T)$ and let $P_{0}$ denote the spectral projection $P_{0}:=(2 \pi i)^{-1} \int_{\Gamma}(\lambda I-T)^{-1} d \lambda$ associated with $\left\{\lambda_{0}\right\}$, via the classical Riesz functional calculus. A classical result shows that the range $P_{0}(X)$ is $N:=H_{0}\left(\lambda_{0} I-T\right)$ (see [24, Proposition 49.1]), whilst ker $P_{0}$ is the analytic core $M:=K\left(\lambda_{0} I-T\right)$ of $\lambda_{0} I-T$ (see [34] and [29]). In this case, $X=M \oplus N$ and

$$
\sigma\left(\lambda_{0} I-T \mid N\right)=\left\{\lambda_{0}\right\}, \quad \sigma\left(\lambda_{0} I-T \mid M\right)=\sigma(T) \backslash\left\{\lambda_{0}\right\},
$$

so $\lambda_{0} I-T \mid M$ is invertible and hence $H_{0}\left(\lambda_{0} I-T \mid M\right)=\{0\}$. Therefore from the decomposition $H_{0}\left(\lambda_{0} I-T\right)=H_{0}\left(\lambda_{0} I-T \mid M\right) \oplus H_{0}\left(\lambda_{0} I-T \mid N\right)$ we deduce that $N=H_{0}\left(\lambda_{0} I-T \mid N\right)$, so $\lambda_{0} I-T \mid N$ is quasi-nilpotent. Hence the pair $(M, N)$ is a GKD for $\lambda_{0} I-T$.

Corollary 2.4. Let $\lambda_{0}$ be an isolated point of $\sigma(T)$. Then

$$
X=H_{0}\left(\lambda_{0} I-T\right) \oplus K\left(\lambda_{0} I-T\right)
$$

and the following assertions are equivalent:

(i) $\lambda_{0} I-T$ is semi-Fredholm;

(ii) $H_{0}\left(\lambda_{0} I-T\right)$ is finite-dimensional;

(iii) $K\left(\lambda_{0} I-T\right)$ is finite-codimensional.

Proof. Since for every operator $T \in L(X)$, both $T$ and $T^{*}$ have SVEP at any isolated point, the equivalence of the assertions easily follows from the decomposition $X=H_{0}\left(\lambda_{0} I-T\right) \oplus K\left(\lambda_{0} I-T\right)$, and from Theorem 2.3.

TheOrem 2.5. Let $T \in L(X)$ and suppose that $T$ or $T^{*}$ has SVEP. Then

$$
\sigma_{\mathrm{ub}}(T)=\sigma_{\mathrm{wa}}(T), \quad \sigma_{\mathrm{lb}}(T)=\sigma_{\mathrm{ws}}(T)
$$

and

$$
\sigma_{\mathrm{b}}(T)=\sigma_{\mathrm{w}}(T) \text {. }
$$

Proof. Suppose first that $T$ has SVEP. To show the first equality of (5) we only need to show the inclusion $\sigma_{\mathrm{ub}}(T) \subseteq \sigma_{\mathrm{wa}}(T)$. If $\lambda \notin \sigma_{\mathrm{wa}}(T)$ then $\lambda I-T \in \Phi_{+}(X)$ and the SVEP implies by Theorem 2.3 that $p(\lambda I-T)<\infty$. Hence $\lambda \notin \sigma_{\mathrm{ub}}(T)$.

Analogously, to prove the equality $\sigma_{\mathrm{lb}}(T)=\sigma_{\mathrm{ws}}(T)$ we only need to show that $\sigma_{\mathrm{lb}}(T) \subseteq \sigma_{\mathrm{ws}}(T)$. If $\lambda \notin \sigma_{\mathrm{ws}}(T)$ then $\lambda I-T \in \Phi_{-}(X)$ with $\beta(\lambda I-T) \leq \alpha(\lambda I-T)$. Again, the SVEP at $\lambda$ entails that $p(\lambda I-T)<\infty$, and hence from Proposition 38.5 of [24] we deduce that $\alpha(\lambda I-T)=\beta(\lambda I-T)$. At this point, the finiteness of $p(\lambda I-T)$ implies by Proposition 38.6 of [24] 
that also $q(\lambda I-T)$ is finite, so $\lambda \notin \sigma_{\mathrm{lb}}(T)$. Therefore $\sigma_{\mathrm{lb}}(T) \subseteq \sigma_{\mathrm{ws}}(T)$ and the proof of the second equality is complete in the case that $T$ has SVEP.

Suppose now that $T^{*}$ has SVEP. Then, by the first part, $\sigma_{\mathrm{ub}}\left(T^{*}\right)=$ $\sigma_{\mathrm{wa}}\left(T^{*}\right)$ and $\sigma_{\mathrm{lb}}\left(T^{*}\right)=\sigma_{\mathrm{ws}}\left(T^{*}\right)$. By duality it follows that $\sigma_{\mathrm{lb}}(T)=\sigma_{\mathrm{ws}}(T)$ and $\sigma_{\mathrm{ub}}(T)=\sigma_{\mathrm{wa}}(T)$. The last equality is clear from the equality $\sigma_{\mathrm{b}}(T)=$ $\sigma_{\mathrm{ub}}(T) \cup \sigma_{\mathrm{lb}}(T)$ and $\sigma_{\mathrm{w}}(T)=\sigma_{\mathrm{wa}}(T) \cup \sigma_{\mathrm{ws}}(T)$.

We shall denote by $\mathcal{H}(\sigma(T))$ the set of all analytic functions defined on a neighborhood of $\sigma(T)$. The next result shows that for operators having SVEP the spectral theorem holds for $\sigma_{\mathrm{w}}(T)$. This is not, in general, true for all operators, whilst the spectral theorem holds for $\sigma_{\mathrm{b}}(T), \sigma_{\mathrm{ub}}(T)$ and $\sigma_{\mathrm{lb}}(T)$ for every $T \in L(X)$ (see [33] or also [1, Chapter 3]).

Corollary 2.6. Suppose that $T$ or $T^{*}$ has $S V E P$ and $f \in \mathcal{H}(\sigma(T))$. Then

$$
\sigma_{\mathrm{wa}}(f(T))=f\left(\sigma_{\mathrm{wa}}(T)\right), \quad \sigma_{\mathrm{ws}}(f(T))=f\left(\sigma_{\mathrm{ws}}(T)\right),
$$

and

$$
\sigma_{\mathrm{w}}(f(T))=f\left(\sigma_{\mathrm{w}}(T)\right) .
$$

Moreover, a-Browder's theorem holds for both $f(T)$ and $f\left(T^{*}\right)$.

Proof. By Theorem 2.5 if $T$ has SVEP (respectively, if $T^{*}$ has SVEP) then $f\left(\sigma_{\mathrm{ub}}(T)\right)=f\left(\sigma_{\mathrm{wa}}(T)\right)$. From the spectral mapping theorem for $\sigma_{\mathrm{ub}}(T)$ we then infer that $f\left(\sigma_{\mathrm{wa}}(T)\right)=\sigma_{\mathrm{ub}}(f(T))$, and again by Theorem 2.5 the last set coincides with $\sigma_{\text {wa }}\left(f(T)\right.$ ), since $f(T)$ (respectively, $f\left(T^{*}\right)=f(T)^{*}$ ) has SVEP by Theorem 3.3.6 of [26]. Hence the first equality of (7) is proved. The second equality of (7) and the equality (8) follow in a similar way.

The argument above shows that if $T$ or $T^{*}$ has SVEP then $a$-Browder's theorem holds for $f(T)$. Moreover, the SVEP for $f(T)$ (respectively, for $\left.f\left(T^{*}\right)\right)$ implies by Theorem 2.5 that $\sigma_{\mathrm{lb}}(f(T))=\sigma_{\mathrm{ws}}(f(T))$, and hence by duality $\sigma_{\mathrm{ub}}\left(f\left(T^{*}\right)\right)=\sigma_{\mathrm{wa}}\left(f\left(T^{*}\right)\right)$, so $a$-Browder's theorem also holds for $f\left(T^{*}\right)$.

Note that Corollary 2.6 extends to a more general situation the result established in Theorem 3.2 of [12]. The spectral theorem for $\sigma_{\mathrm{w}}(T)$ in the case $T$ or $T^{*}$ has SVEP has been proved by using different methods by Curto and Han [11].

An operator $U \in L(X, Y)$ between the Banach spaces $X$ and $Y$ is said to be a quasi-affinity if $U$ is injective and has dense range. The operator $S \in L(Y)$ is said to be a quasi-affine transform of $T \in L(X)$, notation $S \prec T$, if there is a quasi-affinity $U \in L(Y, X)$ such that $T U=U S$. If both $S \prec T$ and $T \prec S$ hold then $S, T$ are called quasi-similar.

Theorem 2.7. If $T \in L(X)$ has $S V E P$ at $\lambda_{0} \in \mathbb{C}$ and $S \in L(Y)$ is a quasi-affine transform of $T$ then $S$ has $S V E P$ at $\lambda_{0}$. In particular, if 
$T \in L(X)$ has $S V E P$ and $S \prec T$ then $f(S)$ satisfies a-Browder's theorem for all $f \in \mathcal{H}(\sigma(T))$.

Proof. Let $f: \mathcal{U} \rightarrow Y$ be an analytic function defined on an open disc $\mathcal{U}$ of $\lambda_{0}$ such that $(\mu I-S) f(\mu)=0$ for all $\mu \in \mathcal{U}$. Then $U(\lambda I-S) f(\mu)=$ $(\mu I-T) U f(\mu)=0$ and the SVEP of $T$ at $\lambda_{0}$ entails that $U f(\mu)=0$ for all $\mu \in \mathcal{U}$. Since $U$ is injective it follows that $f(\mu)=0$ for all $\mu \in \mathcal{U}$, hence $S$ has SVEP at $\lambda_{0}$.

Thus if $T$ has SVEP then $S$ has SVEP. The last assertion is clear by Corollary 2.6.

3. Weyl's theorems for Banach space operators. In this section we give a useful description of operators which satisfy Weyl's theorem, or $a$-Weyl's theorem, in terms of the SVEP. From these characterizations we shall deduce that $a$-Weyl's theorem holds for many classes of Banach space operators.

THEOREM 3.1. If $T \in L(X)$ then the following assertions are equivalent:

(i) Weyl's theorem holds for $T$.

(ii) $T$ has SVEP at every point $\lambda \notin \sigma_{\mathrm{w}}(T)$ and $\pi_{00}(T)=p_{00}(T)$.

In particular, if $T$ or $T^{*}$ has SVEP then Weyl's theorem holds for $T$ if and only if $\pi_{00}(T)=p_{00}(T)$.

Proof. (i) $\Rightarrow$ (ii). Suppose that $T$ satisfies Weyl's theorem. Let $\lambda \notin \sigma_{\mathrm{w}}(T)$. Since $T$ has SVEP at every $\lambda \notin \sigma(T)$ we may assume that $\lambda \in \sigma(T) \backslash \sigma_{\mathrm{w}}(T)$ $=\pi_{00}(T)$. By definition of $\pi_{00}(T)$ we know that $\lambda$ is isolated in $\sigma(T)$, so $T$ has SVEP at $\lambda$.

To show that $\pi_{00}(T)=p_{00}(T)$ it suffices to prove the inclusion $\pi_{00}(T) \subseteq$ $p_{00}(T)$. Suppose that $\lambda \in \pi_{00}(T)=\sigma(T) \backslash \sigma_{\mathrm{w}}(T)$. Since $\lambda I-T$ is Weyl, by Theorem 2.3 it follows that both $p(\lambda I-T)$ and $q(\lambda I-T)$ are finite. Consequently, $\lambda I-T$ is Browder and hence $\lambda \in p_{00}(T)$.

(ii) $\Rightarrow\left(\right.$ i). Let $\lambda \in \sigma(T) \backslash \sigma_{\mathrm{w}}(T)$. By assumption $T$ has SVEP at $\lambda$ and $\lambda I-T$ is Weyl, so by Theorem 2.3 both $p(\lambda I-T)$ and $q(\lambda I-T)$ are finite. This shows that

$$
\sigma(T) \backslash \sigma_{\mathrm{w}}(T) \subseteq p_{00}(T)=\pi_{00}(T) .
$$

On the other hand, if $\lambda \in \pi_{00}(T)=p_{00}(T)$ then $p(\lambda I-T)=q(\lambda I-T)<\infty$ and $\alpha(\lambda I-T)<\infty$, so by Proposition 38.6 of [24] we have $\beta(\lambda I-T)=$ $\alpha(\lambda I-T)<\infty$. Hence $\lambda \in \sigma(T) \backslash \sigma_{\mathrm{w}}(T)$. Therefore $\sigma(T) \backslash \sigma_{\mathrm{w}}(T)=\pi_{00}(T)$. The last assertion is clear in the case $T$ has SVEP. Suppose that $T^{*}$ has SVEP and $\pi_{00}(T)=p_{00}(T)$. By Theorem 2.5, $\sigma_{\mathrm{w}}(T)=\sigma_{\mathrm{b}}(T)$, and hence

$$
\pi_{00}(T)=p_{00}(T)=\sigma(T) \backslash \sigma_{\mathrm{b}}(T)=\sigma(T) \backslash \sigma_{\mathrm{w}}(T),
$$

so the proof is complete 
In general, we cannot expect that Weyl's theorem holds for operators $T$ for which $T$ or $T^{*}$ has SVEP. For instance, if $T \in L\left(\ell^{2}(\mathbb{N})\right)$ is defined by

$$
T\left(x_{0}, x_{1}, \ldots\right):=\left(\frac{1}{2} x_{1}, \frac{1}{3} x_{2}, \ldots\right) \quad \text { for all }\left(x_{n}\right) \in \ell^{2}(\mathbb{N}),
$$

then $T$ is quasi-nilpotent and hence both $T$ and $T^{*}$ have SVEP. But $T$ does not satisfy Weyl's theorem, since $p_{00}(T)=\emptyset$, whilst $\pi_{00}(T)=\{0\}$.

Definition 3.2. A bounded operator $T \in L(X)$ on a Banach space $X$ is said to have property $\left(H_{p}\right)$ if for every $\lambda \in \mathbb{C}$ there exists an integer $p:=p(\lambda) \geq 1$ such that

$$
H_{0}(\lambda I-T)=\operatorname{ker}(\lambda I-T)^{p} .
$$

A bounded operator $T \in L(X)$ is said to be isoloid if every isolated point of $\sigma(T)$ is an eigenvalue of $T$.

Lemma 3.3. If $T \in L(X)$ has property $\left(H_{p}\right)$ then $T$ has $S V E P$ and every isolated point of the spectrum is a pole of the resolvent. In particular, $T$ is isoloid.

Proof. $T$ has SVEP by Theorem 1.6 of [3]. Furthermore, if $\lambda \in$ iso $\sigma(T)$ then by Theorem 2.4 we have

$$
X=H_{0}(\lambda I-T) \oplus K(\lambda I-T)=\operatorname{ker}(\lambda I-T)^{p} \oplus K(\lambda I-T),
$$

and consequently

$$
(\lambda I-T)^{p}(X)=(\lambda I-T)^{p}(K(\lambda I-T))=K(\lambda I-T) .
$$

Therefore $X=\operatorname{ker}(\lambda I-T)^{p} \oplus(\lambda I-T)^{p}(X)$, from which it follows by Proposition 38.4 of [24] that $p(\lambda I-T)=q(\lambda I-T) \leq p$, i.e. $\lambda$ is a pole of the resolvent of $T$.

We owe the following result to a recent work of M. Oudghiri [30].

TheOREm 3.4. Let $T \in L(X), X$ a Banach space, and suppose that there exists an analytic function $h \in \mathcal{H}(\sigma(T))$ with domain $\mathcal{U}$, not identically constant in any component of $\mathcal{U}$, such that $h(T)$ has property $\left(H_{p}\right)$. Then Weyl's theorem holds for both $f(T)$ and $f\left(T^{*}\right)$ for every $f \in \mathcal{H}(\sigma(T))$. In particular, if $T$ has property $\left(H_{p}\right)$ then Weyl's theorem holds for both $T$ and $T^{*}$.

The class of operators having property $\left(H_{p}\right)$ is rather large. In fact, as observed in [30], every generalized scalar operator and every subscalar operator on a Banach space has property $\left(H_{p}\right)$ (see [30] and [26] for relevant definitions). In particular, from Theorem 3.4 one may deduce that Weyl's theorem holds for the following classes of operators:

(a) An operator $T \in L(H), H$ a Hilbert space, is called log-hyponormal if $T$ is invertible and satisfies $\log \left(T^{*} T\right) \geq \log \left(T T^{*}\right)$. Every $\log$-hyponormal 
operator has property $\left(H_{1}\right)$ (see [7]), so Weyl's theorem holds for this class of operators (see also [9]).

(b) An operator $T \in L(H)$ on a Hilbert space $H$ is called p-hyponormal, with $0<p \leq 1$, if $\left(T^{*} T\right)^{p} \geq\left(T T^{*}\right)^{p}$. Every $p$-hyponormal has property $\left(H_{p}\right)$, since it is subscalar [28]. Weyl's theorem holds for operators on Hilbert spaces for which either $T$ or $T^{*}$ is a $p$-hyponormal operator [8].

(c) An operator $T \in L(H)$ is said to be $M$-hyponormal if there is $M>0$ for which $T T^{*} \leq M T^{*} T$. Also every $M$-hyponormal operator $T$ obeys Weyl's theorem since it is subscalar [28].

(d) An operator $T \in L(H)$ is said to be $*$-paranormal if $\left\|T^{*} x\right\|^{2} \leq\left\|T^{2} x\right\|$ for every unit vector $x \in H$. If $\lambda I-T$ is $*$-paranormal for every $\lambda \in \mathbb{C}$ then $T$ is said to be totally $*$-paranormal. If $T$ is totally $*$-paranormal then $T$ has property $\left(H_{1}\right)$ (see [22, Lemma 2.2]).

(e) An important class of operators having property $\left(H_{1}\right)$ is given by the class of all multipliers of commutative semi-simple Banach algebras [3]. In particular, every convolution operator on the group algebra $L^{1}(G), G$ a locally compact abelian group, has property $\left(H_{1}\right)$.

Also transaloid operators on Banach spaces have property $\left(H_{1}\right)[11$, Theorem 2.3].

For a bounded operator $T \in L(X)$ on a Banach space $X$ define

$$
p_{00}^{\mathrm{a}}(T):=\sigma_{\mathrm{a}}(T) \backslash \sigma_{\mathrm{ub}}(T)=\left\{\lambda \in \sigma_{\mathrm{a}}(T): \lambda I-T \in \mathcal{B}_{+}(X)\right\} .
$$

We have

$$
p_{00}^{\mathrm{a}}(T) \subseteq \pi_{00}^{\mathrm{a}}(T) \quad \text { for every } T \in L(X) .
$$

In fact, if $\lambda \in p_{00}^{\mathrm{a}}(T)$ then $\lambda I-T \in \Phi_{+}(X)$ and $p(\lambda I-T)<\infty$. By Theorem 2.3, $\lambda$ is isolated in $\sigma_{\mathrm{a}}(T)$. Furthermore, $0<\alpha(\lambda I-T)<\infty$ since $(\lambda I-T)(X)$ is closed and $\lambda \in \sigma_{\mathrm{a}}(T)$.

Theorem 3.5. If $T \in L(X)$ the following statements are equivalent:

(i) $T$ satisfies a-Weyl's theorem;

(ii) $T$ has SVEP at every point $\lambda \notin \sigma_{\mathrm{wa}}(T)$ and $p_{00}^{\mathrm{a}}(T)=\pi_{00}^{\mathrm{a}}(T)$.

In particular, if $T$ or $T^{*}$ has SVEP, then a-Weyl's theorem holds for $T$ if and only if $p_{00}^{\mathrm{a}}(T)=\pi_{00}^{\mathrm{a}}(T)$.

Proof. (i) $\Rightarrow$ (ii). Suppose that $T$ satisfies $a$-Weyl's theorem. Let $\lambda \notin$ $\sigma_{\text {wa }}(T)$. To show that $T$ has SVEP at $\lambda$ we may assume, since $T$ has SVEP at every point $\lambda \notin \sigma_{\mathrm{a}}(T)$, that $\lambda \in \sigma_{\mathrm{a}}(T) \backslash \sigma_{\mathrm{wa}}(T)=\pi_{00}^{\mathrm{a}}(T)$. Since $\lambda$ is isolated in $\sigma_{\mathrm{a}}(T)$ it follows that $T$ has SVEP at $\lambda$. To prove that $p_{00}^{\mathrm{a}}(T)=\pi_{00}^{\mathrm{a}}(T)$ it suffices to prove $\pi_{00}^{\mathrm{a}}(T) \subseteq p_{00}^{\mathrm{a}}(T)$. Let $\lambda \in \pi_{00}^{\mathrm{a}}(T)=\sigma_{\mathrm{a}}(T) \backslash \sigma_{\mathrm{wa}}(T)$. Then $\lambda I-T \in \Phi_{+}(X)$ and since $\lambda$ is isolated in $\sigma_{\mathrm{a}}(T)$ it follows by Theorem 2.3 that $p(\lambda I-T)<\infty$. Hence $\lambda \in p_{00}^{\mathrm{a}}(T)$, and consequently $\pi_{00}^{\mathrm{a}}(T) \subseteq p_{00}^{\mathrm{a}}(T)$. 
(ii) $\Rightarrow$ (i). Let $\lambda \in \sigma_{\mathrm{a}}(T) \backslash \sigma_{\mathrm{wa}}(T)$. Then $T$ has SVEP at $\lambda$ and $\lambda I-T \in$ $\Phi_{+}(X)$, so by Theorem 2.3 the ascent $p(\lambda I-T)$ is finite. This shows that

$$
\sigma_{\mathrm{a}}(T) \backslash \sigma_{\mathrm{wa}}(T) \subseteq p_{00}^{\mathrm{a}}(T)=\pi_{00}^{\mathrm{a}}(T) .
$$

On the other hand, if $\lambda \in \pi_{00}^{\mathrm{a}}(T)=p_{00}^{\mathrm{a}}(T)$ then $\lambda I-T \in \Phi_{+}(X)$ with $p(\lambda I-T)<\infty$. From Proposition 38.5 of [24] we deduce that $\alpha(\lambda I-T)$ $\leq \beta(\lambda I-T)$, so $\operatorname{ind}(\lambda I-T) \leq 0$. Therefore, $\lambda \in \sigma_{\mathrm{a}}(T) \backslash \sigma_{\mathrm{wa}}(T)$ and consequently $\sigma_{\mathrm{a}}(T) \backslash \sigma_{\mathrm{wa}}(T)=\pi_{00}^{\mathrm{a}}(T)$.

The last assertion is clear in the case where $T$ has SVEP. Suppose that $T^{*}$ has SVEP. If $a$-Weyl's theorem holds for $T$ then $p_{00}^{\mathrm{a}}(T)=\pi_{00}^{\mathrm{a}}(T)$ by the first part of the proof. Conversely, suppose that $p_{00}^{\mathrm{a}}(T)=\pi_{00}^{\mathrm{a}}(T)$. The SVEP for $T^{*}$ ensures by Theorem 2.5 that $\sigma_{\mathrm{wa}}(T)=\sigma_{\mathrm{ub}}(T)$, so

$$
\pi_{00}^{\mathrm{a}}(T)=p_{00}^{\mathrm{a}}(T)=\sigma_{\mathrm{a}}(T) \backslash \sigma_{\mathrm{ub}}(T)=\sigma_{\mathrm{a}}(T) \backslash \sigma_{\mathrm{wa}}(T),
$$

and hence $a$-Weyl's theorem holds for $T$ also in the case where $T^{*}$ has SVEP.

The next result has a crucial role in proving that many classes of operators satisfy $a$-Weyl's theorem.

TheOREM 3.6. If $T \in L(X)$ has SVEP then the following statements are equivalent:

(i) Weyl's theorem holds for T*;

(ii) a-Weyl's theorem holds for $T^{*}$.

Analogously, if the dual $T^{*}$ of $T$ has SVEP then the following statements are equivalent:

(iii) Weyl's theorem holds for T;

(iv) $a$-Weyl's theorem holds for $T$.

Proof. (i) $\Leftrightarrow$ (ii). We only have to show the implication (i) $\Rightarrow$ (ii). Suppose that $T^{*}$ satisfies Weyl's theorem, i.e., $\sigma\left(T^{*}\right) \backslash \sigma_{\mathrm{w}}\left(T^{*}\right)=\pi_{00}\left(T^{*}\right)$. Since $T$ has SVEP we have $\sigma_{\mathrm{a}}\left(T^{*}\right)=\sigma\left(T^{*}\right)$ (see [26, Proposition 1.3.2]), hence $\pi_{00}^{\mathrm{a}}\left(T^{*}\right)=$ $\pi_{00}\left(T^{*}\right)$. The SVEP for $T$ also implies by Theorem 2.5 and [2, Corollary 2.8] that

$$
\sigma_{\mathrm{w}}(T)=\sigma_{\mathrm{b}}(T)=\sigma_{\mathrm{lb}}(T)=\sigma_{\mathrm{ws}}(T) .
$$

By duality we then obtain $\sigma_{\mathrm{w}}\left(T^{*}\right)=\sigma_{\mathrm{wa}}\left(T^{*}\right)$, so

$$
\pi_{00}^{\mathrm{a}}\left(T^{*}\right)=\pi_{00}\left(T^{*}\right)=\sigma\left(T^{*}\right) \backslash \sigma_{\mathrm{w}}\left(T^{*}\right)=\sigma_{\mathrm{a}}\left(T^{*}\right) \backslash \sigma_{\mathrm{wa}}\left(T^{*}\right),
$$

and hence $a$-Weyl's theorem holds for $T^{*}$.

To prove the equivalence (iii) $\Leftrightarrow$ (iv) we proceed in a similar way. Suppose that the dual $T^{*}$ has SVEP and that Weyl's theorem holds for $T$. Then $\sigma(T) \backslash \sigma_{\mathrm{w}}(T)=\pi_{00}(T)$ and by [26, Proposition 1.3.2], $\sigma_{\mathrm{a}}(T)=\sigma(T)$, so that 
$\pi_{00}^{\mathrm{a}}(T)=\pi_{00}(T)$. By Theorem 2.5 and $[2$, Corollary 2.8] we have

$$
\sigma_{\mathrm{w}}(T)=\sigma_{\mathrm{b}}(T)=\sigma_{\mathrm{ub}}(T)=\sigma_{\mathrm{wa}}(T) .
$$

From this it follows that

$$
\pi_{00}^{\mathrm{a}}(T)=\pi_{00}(T)=\sigma(T) \backslash \sigma_{\mathrm{w}}(T)=\sigma_{\mathrm{a}}(T) \backslash \sigma_{\mathrm{wa}}(T),
$$

so $a$-Weyl's theorem holds for $T$.

In what follows, we shall denote by $M^{\perp}$, for every $M \subset X$, the annihilator of $M \subseteq X$, and by ${ }^{\perp} N$ the pre-annihilator of $N \subseteq X^{*}$. The next result improves Theorem 3.4.

TheOREm 3.7. If $T \in L(X)$ has property $\left(H_{p}\right)$ then a-Weyl's holds for $f\left(T^{*}\right)$ for every $f \in \mathcal{H}(\sigma(T))$. Analogously, if $T^{*}$ has property $\left(H_{p}\right)$ then $a$-Weyl's holds for $f(T)$ for every $f \in \mathcal{H}(\sigma(T))$.

Proof. If $T \in L(H)$ has property $\left(H_{p}\right)$ then $T$ has SVEP by Lemma 3.3, and hence by Theorem 3.3.6 of [26], $f(T)$ has SVEP for every $f \in \mathcal{H}(\sigma(T))$. Moreover, by Theorem 3.4 Weyl's theorem holds for $f(T)^{*}=f\left(T^{*}\right)$, and this by Theorem 3.6 is equivalent to saying that $a$-Weyl's theorem holds for $f\left(T^{*}\right)$.

Suppose now that $T^{*}$ has property $\left(H_{p}\right)$. We show first that Weyl's theorem holds for $T$. We know that $T^{*}$ has SVEP, again by Lemma 3.3, so, in order to show that $T$ satisfies Weyl's theorem it suffices by Theorem 3.1 to prove that $\pi_{00}(T)=p_{00}(T)$. Let $\lambda \in \pi_{00}(T)$. Then $\lambda$ is an isolated point in $\sigma(T)=\sigma\left(T^{*}\right)$, and hence by Lemma $3.3, \lambda$ is a pole of the resolvent of $T^{*}$, i.e. $p:=p\left(\lambda I^{*}-T^{*}\right)=q\left(\lambda I^{*}-T^{*}\right)<\infty$. Therefore, $X^{*}=\operatorname{ker}\left(\lambda I^{*}-T^{*}\right)^{p} \oplus$ $\left(\lambda I^{*}-T^{*}\right)^{p}\left(X^{*}\right)$ and since $\left(\lambda I^{*}-T^{*}\right)^{p}\left(X^{*}\right)$ is closed it follows that also $(\lambda I-T)^{p}(X)$ is closed. By the classical closed range theorem we then have $X={ }^{\perp} \operatorname{ker}\left(\lambda I^{*}-T^{*}\right)^{p} \oplus{ }^{\perp}\left(\lambda I^{*}-T^{*}\right)^{p}\left(X^{*}\right)=\operatorname{ker}(\lambda I-T)^{p} \oplus(\lambda I-T)^{p}(X)$, so by Proposition 38.4 of [24] we conclude that $p(\lambda I-T)=q(\lambda I-T)<\infty$. Finally, $\alpha(\lambda I-T)<\infty$ by assumption and consequently $\beta(\lambda I-T)<\infty$, from which we conclude that $\lambda \in p_{00}(T)$. Hence Weyl's theorem holds for $T$.

The argument above shows that if $T^{*}$ has property $\left(H_{p}\right)$ then $T$ is isoloid. We prove now that Weyl's theorem holds for $f(T)$. In fact, since $T$ is isoloid and $T$ satisfies Weyl's theorem, by [27, Lemma] we have

$$
\sigma(f(T)) \backslash \pi_{00}(f(T))=f\left(\sigma(T) \backslash \pi_{00}(T)\right)=f\left(\sigma_{\mathrm{w}}(T)\right) .
$$

The SVEP for $T^{*}$ entails that $f\left(\sigma_{\mathrm{w}}(T)\right)=\sigma_{\mathrm{w}}(f(T))$, by Theorem 2.5, and hence Weyl's theorem holds for $f(T)$.

Remark 3.8. Theorem 3.7 implies that if $T$ is a multiplier of a commutative semi-simple Banach algebra, or if $T$ is transaloid, then $a$-Weyl's theorem holds for $f\left(T^{*}\right)$ for all $f \in \mathcal{H}(\sigma(T))$. 
REMARK 3.9. The following example shows that property $\left(H_{p}\right)$ for an operator $T \in L(X)$ does not imply, in general, that $T$ satisfies $a$-Weyl's theorem. Let $T$ be the hyponormal operator $T$ given by the direct sum of the 1-dimensional zero operator and the unilateral right shift $R$ on $\ell^{2}(\mathbb{N})$. Then 0 is an isolated point of $\sigma_{\mathrm{a}}(T)$ and $0 \in \pi_{00}^{\mathrm{a}}(T)$, whilst $0 \notin p_{00}^{\mathrm{a}}(T)$, since $p(T)=p(R)=\infty$. Hence, by Theorem 3.5, $T$ does not satisfy $a$-Weyl's theorem.

Corollary 3.10. Let $T \in L(X), X$ a Banach space, be a generalized scalar operator. Then a-Weyl's theorem holds for $f(T)$ and $f\left(T^{*}\right)$ for all $f \in \mathcal{H}(\sigma(T))$.

Proof. Every generalized scalar operator has property $\left(H_{p}\right)$, so by Theorem 3.7 Weyl's theorem holds for $f(T)$ and $f\left(T^{*}\right)$. Furthermore, since $T$ is decomposable [26] both $T$ and $T^{*}$ have SVEP, and consequently both $f(T)$ and $f\left(T^{*}\right)$ have SVEP. By Theorem 3.6 we then conclude that $a$-Weyl's theorem holds for $f(T)$ and $f\left(T^{*}\right)$.

TheOREM 3.11. Suppose that $T \in L(X)$ has property $(\beta)$ and $S \in L(Y)$ has property $(\delta)$. If $T$ and $S$ are quasi-similar then the following statements are equivalent:

(i) T satisfies Weyl's theorem;

(ii) $S$ satisfies a-Weyl's theorem.

Proof. Since $T$ has property $(\beta)$ we have $\sigma(T)=\sigma(S)$ by a result of Putinar [31], so iso $\sigma(T)=$ iso $\sigma(S)$. Moreover, property $(\beta)$ entails that $T$ has SVEP and hence also $S$ has SVEP, by Theorem 2.7. From Theorem 5 of [16], $T$ satisfies Weyl's theorem precisely when $S$ satisfies Weyl's theorem. Since property $(\delta)$ for $S$ entails that $S^{*}$ has SVEP, by Theorem 3.6 we conclude that (i) and (ii) are equivalent.

Corollary 3.12. Suppose that two quasi-similar operators $T \in L(X)$ and $S \in L(Y)$ are decomposable. Then $T$ satisfies $a$-Weyl's theorem if and only if $S$ does. In particular, every decomposable operator quasi-similar to a generalized scalar operator satisfies a-Weyl's theorem.

Proof. If $T$ is decomposable then $T^{*}$ has SVEP, so $a$-Weyl's theorem and Weyl's theorem for $T$ are equivalent. The statements are then clear from Theorem 3.11 and Corollary 3.10.

4. Algebraically paranormal operators. In this section we shall denote by $H$ a complex infinite-dimensional Hilbert space. In the case of operators defined on Hilbert spaces instead of the dual $T^{*}$ it is more appropriate to consider the Hilbert adjoint $T^{\prime}$ of $T \in L(H)$. However, some of the basic results established in the previous section for $T^{*}$ are also true for the adjoint $T^{\prime}$. In fact, by means of the classical Fréchet-Riesz representation theorem 
we know that if $U$ is the conjugate-linear isometry that associates to each $y \in H$ the linear form $x \mapsto\langle x, y\rangle$ then $U T^{\prime}=T^{*} U$. From this equality and from Theorem 2.3 it easily follows that

$$
q(\lambda I-T)<\infty \Rightarrow T^{\prime} \text { has SVEP at } \lambda .
$$

Note that $\sigma_{\mathrm{w}}\left(T^{\prime}\right)=\overline{\sigma_{\mathrm{w}}(T)}$. Furthermore, using an argument similar to that in the proof of Theorem 2.7, from the equality $U T^{\prime}=T^{*} U$ we easily deduce that

$$
T^{\prime} \text { has SVEP at } \lambda_{0} \Leftrightarrow T^{*} \text { has SVEP at } \lambda_{0} \text {. }
$$

Hence the SVEP of $T^{\prime}$ ensures by Corollary 2.6 that the equality $f\left(\sigma_{\mathrm{w}}(T)\right)=$ $f\left(\sigma_{\mathrm{w}}(T)\right)$ holds for all $f \in \mathcal{H}(\sigma(T))$.

TheOREM 4.1. If $T^{\prime}$ has property $\left(H_{p}\right)$ and $f \in \mathcal{H}(\sigma(T))$ then a-Weyl's theorem holds for $f(T)$.

Proof. It is easily seen that if $T^{\prime}$ has property $\left(H_{p}\right)$ then also $T^{*}$ has property $\left(H_{p}\right)$ (this property is preserved by quasi-affine transformations, and the same argument of [30, Lemma 3.2] works in our case, since $U T^{\prime}=$ $T^{*} U$ and $U$ is an isometry). By Theorem 3.7 it then follows that $a$-Weyl's theorem holds for $f(T)$.

REMARK 4.2. It should be noted that Theorem 4.1 provides a general framework for $a$-Weyl's theorem, from which all the results listed in the sequel follow as special cases. Note that in the literature $a$-Weyl's theorem has been proved separately for each class of operators.

(i) If $T^{\prime}$ is log-hyponormal or p-hyponormal then a-Weyl's theorem holds for $f(T)$ [15, Theorem 3.3], [17, Theorem 4.2].

(ii) If $T^{\prime}$ is $M$-hyponormal then a-Weyl's theorem holds for $f(T)[15$, Theorem 3.6].

(iii) If $T^{\prime}$ is totally $*$-paranormal then a-Weyl's theorem holds for $f(T)$ [22, Theorem 2.10].

A bounded operator $T \in L(X)$ on a Banach space $X$ is said to be paranormal if

$$
\|T x\|^{2} \leq\left\|T^{2} x\right\|\|x\| \quad \text { for all } x \in X .
$$

$T \in L(X)$ is called totally paranormal if $\lambda I-T$ is paranormal for all $\lambda \in \mathbb{C}$. Every totally paranormal $T$ operator satisfies condition $\left(H_{1}\right)$ (see [7]), and hence Weyl's theorem holds for T. By Theorem 4.1 we also have

(iv) If $T^{\prime} \in L(H)$ is totally paranormal then a-Weyl's theorem holds for $f(T)$.

Theorem 3.4 and Theorem 4.1 do not work for paranormal operators. In fact, these operators do not have property $\left(H_{p}\right)$ (see Remark following 
Lemma 3 in [18]). However, we shall see that Weyl's theorem for paranormal operators may be deduced from Theorem 3.1.

Every paranormal operator on a Hilbert space has SVEP. To see this note first that for these operators we have $\operatorname{ker}(\lambda I-T) \subseteq \operatorname{ker}\left(\lambda I-T^{\prime}\right)$ for all $\lambda \in \mathbb{C}$ and from this it easily follows that $p(\lambda I-T) \leq 1$ for all $\lambda \in \mathbb{C}$, so $T$ has SVEP (see also [18]). Observe that every paranormal operator $T$ is normaloid (i.e. $\|T\|=r(T)$, the spectral radius of $T$, see [24, Proposition 54.6]), so if $T$ is quasi-nilpotent then $T=0$.

An operator $T \in L(X)$ for which there exists a complex nonconstant polynomial $h$ such that $h(T)$ is paranormal is said to be algebraically paranormal. Note that algebraic paranormality is preserved under translation by scalars and under restriction to closed invariant subspaces.

LEMma 4.3. If $T \in L(H)$ is algebraically paranormal then $T$ has SVEP and every isolated point of the spectrum is a pole of the resolvent. In particular, both $T$ and $T^{\prime}$ are isoloid.

Proof. Let $h$ be a nonconstant complex polynomial such that $h(T)$ is paranormal. Then $h(T)$ has SVEP and hence by Theorem 3.3.9 of [26] also $T$ has SVEP. To prove the second assertion note first that every quasi-nilpotent algebraically paranormal operator $T$ is nilpotent. In fact, $\sigma(h(T))=h(\sigma(T))$ $=\{h(0)\}$, so $h(0) I-h(T)$ is quasi-nilpotent. Since $h(0) I-T$ is paranormal, there is some $n \in \mathbb{N}$ such that

$$
0=h(0) I-h(T)=a T^{m} \prod_{i=1}^{n}\left(\lambda_{i} I-T\right) \quad \text { with } \lambda_{i} \neq 0 .
$$

Since all $\lambda_{i} I-T$ are invertible it follows that $T^{m}=0$.

Now, if $\lambda \in$ iso $\sigma(T), M:=K(\lambda I-T)$ and $N:=H_{0}(\lambda I-T)$ then $(M, N)$ is a GKD for $\lambda I-T$. Since $\lambda I-T \mid N$ is quasi-nilpotent and algebraically paranormal it follows that $\lambda I-T \mid N$ is nilpotent and hence $\lambda I-T$ is of Kato type. The SVEP for $T$ and $T^{\prime}$ at $\lambda$ then implies, by Theorem 2.3 , that both $p(\lambda I-T)$ and $q(\lambda I-T)$ are finite. Hence $\lambda$ is a pole of the resolvent of $T$. This implies that $T$ is isoloid.

Analogously, to prove that $T^{\prime}$ is isoloid we prove that every isolated point of $\sigma\left(T^{\prime}\right)$ is a pole of the resolvent of $T^{\prime}$. Let $\lambda$ be an isolated point in $\sigma\left(T^{\prime}\right)=\overline{\sigma(T)}$. Then $\bar{\lambda}$ is isolated in $\sigma(T)$, and hence by the first part of the proof the point $\bar{\lambda}$ is a pole of the resolvent of $T$, hence $p:=p(\bar{\lambda} I-T)=$ $q(\bar{\lambda} I-T)<\infty$. Consequently, $H=\operatorname{ker}(\bar{\lambda} I-T)^{p} \oplus(\bar{\lambda} I-T)^{p}(X)$ and the range $(\bar{\lambda} I-T)^{p}(X)$ is closed. From this it follows that $H=\left(\operatorname{ker}(\bar{\lambda} I-T)^{p}\right)^{\perp} \oplus\left((\bar{\lambda} I-T)^{p}(H)\right)^{\perp}=\left(\lambda I-T^{\prime}\right)^{p}(H) \oplus \operatorname{ker}\left(\lambda I-T^{\prime}\right)^{p}$, where now $N^{\perp}$ denotes the orthogonal of $N \subseteq H$. Therefore $p\left(\lambda I-T^{\prime}\right)=$ $q\left(\lambda I-T^{\prime}\right)<\infty$, or equivalently $\lambda$ is a pole of the resolvent of $T^{\prime}$. 
The next result improves Corollary 4 of [23] and Theorem 2.4 of [12].

TheOrEm 4.4. Let $T \in L(H)$. Then the following statements hold:

(i) If $T \in L(H)$ is algebraically paranormal then Weyl's theorem holds for $f(T)$ for all $f \in \mathcal{H}(\sigma(T))$.

(ii) If $T^{\prime}$ is algebraically paranormal then a-Weyl's theorem holds for $f(T)$ for all $f \in \mathcal{H}(\sigma(T))$.

Proof. (i) Suppose that $T$ is algebraically paranormal. We show first that Weyl's theorem holds for $T$. Since $T$ has SVEP it suffices by Theorem 3.1 to show that $p_{00}(T)=\pi_{00}(T)$. Suppose that $\lambda \in \pi_{00}(T)$. By assumption $\alpha(\lambda I-T)<\infty$ and $\lambda$ is isolated in $\sigma(T)$, so, by Lemma 4.3, $\lambda$ is a pole and hence $p(\lambda I-T)=q(\lambda I-T)<\infty$. From [24, Proposition 38.6] it then follows that $\beta(\lambda I-T)<\infty$, i.e. $\lambda \in p_{00}(T)$. Therefore Weyl's theorem holds for $T$.

To show that Weyl's theorem holds for $f(T)$ note that, $T$ being isoloid, by $[27$, Lemma] we have

$$
\sigma(f(T)) \backslash \pi_{00}(f(T))=f\left(\sigma(T) \backslash \pi_{00}(T)\right)=f\left(\sigma_{\mathrm{w}}(T)\right),
$$

where the last equality holds since $T$ satisfies Weyl's theorem. By Corollary 2.6 the SVEP for $T$ implies that $f\left(\sigma_{\mathrm{w}}(T)\right)=\sigma_{\mathrm{w}}(f(T))$, and consequently

$$
\sigma(f(T)) \backslash \pi_{00}(f(T))=\sigma_{\mathrm{w}}(f(T)),
$$

so Weyl's theorem holds for $f(T)$.

(ii) Suppose now that $T^{\prime}$ is algebraically paranormal. We show first that Weyl's theorem holds for $T$. Since $T^{\prime}$ is algebraically paranormal it follows that $T^{\prime}$, and hence also $T^{*}$, has SVEP. In order to show that $T$ satisfies Weyl's theorem it then suffices, by Theorem 3.1, to prove that $\pi_{00}(T)=$ $p_{00}(T)$.

Let $\lambda \in \pi_{00}(T)$. Then $\lambda$ is an isolated point in $\sigma(T)=\overline{\sigma\left(T^{\prime}\right)}$, and hence by Lemma $4.3, \bar{\lambda}$ is a pole of the resolvent of $T^{\prime}$, i.e. $p:=p\left(\bar{\lambda} I-T^{\prime}\right)=$ $q\left(\bar{\lambda} I-T^{\prime}\right)<\infty$. We have $H=\operatorname{ker}\left(\bar{\lambda} I-T^{\prime}\right)^{p} \oplus\left(\bar{\lambda} I-T^{\prime}\right)^{p}(H)$ and since $\left(\bar{\lambda} I-T^{\prime}\right)^{p}(H)$ is closed it follows that $(\lambda I-T)^{p}(H)$ is closed. We also have $H=\left(\operatorname{ker}\left(\bar{\lambda} I-T^{\prime}\right)^{p}\right)^{\perp} \oplus\left(\left(\bar{\lambda} I-T^{\prime}\right)^{p}(H)\right)^{\perp}=(\lambda I-T)^{p}(H) \oplus \operatorname{ker}(\lambda I-T)^{p}$, and again by Proposition 38.4 of [24] we conclude that $p(\lambda I-T)=q(\lambda I-T)$ $<\infty$, i.e. $\lambda$ is a pole of the resolvent of $T$.

Finally, $\alpha(\lambda I-T)<\infty$ by assumption and consequently $\beta(\lambda I-T)<\infty$, from which we conclude that $\lambda \in p_{00}(T)$. Hence Weyl's theorem holds for $T$.

The argument above also proves that if $T^{\prime}$ is algebraically paranormal then $T$ is isoloid. Since the SVEP for $T^{\prime}$ implies $f\left(\sigma_{\mathrm{w}}(T)\right)=\sigma_{\mathrm{w}}(f(T))$, arguing as in the proof of part (i) it readily follows that Weyl's theorem holds for $f(T)$ for all $f \in \mathcal{H}(\sigma(T))$. 
Finally, since $T^{\prime}$ has SVEP, so does $f\left(T^{\prime}\right)$, and hence also $f\left(T^{*}\right)$. By Theorem 3.6 it follows that $a$-Weyl's theorem holds for $f(T)$.

Notice that Theorem 4.4 implies Weyl's theorem for paranormal operators. Weyl's theorem for an algebraically paranormal operator has been established by Curto and Han [12] by using different methods. Since every $p$-hyponormal operator is paranormal, Weyl's theorem for $p$-hyponormal operators ([8]) and algebraically hyponormal operators ([23]) may also be deduced from Theorem 4.4 .

The operator defined in Remark 3.9 shows that, in general, we cannot expect that $a$-Weyl's theorem holds for any algebraically paranormal operator.

A bounded operator $T \in L(H)$ is said to be quasi-hyponormal if $\left\|T^{*} T x\right\|$ $\leq\left\|T^{2} x\right\|$ for all $x \in H$. Every quasi-hyponormal operator is paranormal [21], so part (ii) of Theorem 4.4 subsumes the following result of S. V. Djordjević and D. S. Djordjević [14, Theorem 3.4] and improves Corollary 5.7 of D. S. Djordjević [13].

COROllary 4.5. If $T^{\prime} \in L(H)$ is quasi-hyponormal then a-Weyl's theorem holds for $f(T)$ for every $f \in \mathcal{H}(\sigma(T))$.

\section{References}

[1] P. Aiena, Fredholm and Local Spectral Theory, with Application to Multipliers, Kluwer, 2004.

[2] P. Aiena and C. Carpintero, Single valued extension property and semi-Browder spectra, Acta Sci. Math. (Szeged) 70 (2004), 265-278.

[3] P. Aiena, M. L. Colasante and M. Gonzalez, Operators which have a closed quasinilpotent part, Proc. Amer. Math. Soc. 130 (2002), 2701-2710.

[4] P. Aiena, T. L. Miller and M. M. Neumann, On a localized single-valued extension property, Math. Proc. Roy. Irish Acad. Sect. A 104 (2004), 17-34.

[5] P. Aiena and O. Monsalve, The single valued extension property and the generalized Kato decomposition property, Acta Sci. Math. (Szeged) 67 (2001), 461-477.

[6] P. Aiena and E. Rosas, The single valued extension property at the points of the approximate point spectrum, J. Math. Anal. Appl. 279 (2003), 180-188.

[7] P. Aiena and F. Villafañe, Weyl's theorem for some classes of operators, Integral Equations Operator Theory, to appear.

[8] M. Chō, M. Itoh and S. Ōshiro, Weyl's theorems holds for p-hyponormal operators, Glasgow Math. J. 39 (1997), 217-220.

[9] M. Chō, I. H. Jeon and J. I. Lee, Spectral and structural properties of log-hyponormal operators, ibid. 42 (2000), 345-350.

[10] L. A. Coburn, Weyl's theorem for nonnormal operators, Michigan Math. J. 13 (1966), 285-288.

[11] R. E. Curto and Y. M. Han, Weyl's theorem, a-Weyl's theorem, and local spectral theory, J. London Math. Soc. (2) 67 (2003), 499-509.

[12] - - - Weyl's theorem for algebraically paranormal operators, Integral Equations Operator Theory 50 (2004), 169-196. 
[13] D. S. Djordjević, Operators obeying a-Weyl's theorem, Publ. Math. Debrecen 55 (1999), 283-298.

[14] S. V. Djordjević and D. S. Djordjević, Weyl's theorems: continuity of the spectrum and quasi-hyponormal operators, Acta Sci. Math (Szeged) 64, no. 3 (1998), 259-269.

[15] S. V. Djordjević and Y. M. Han, A note on a-Weyl's theorem, J. Math. Anal. Appl. 260, no. 8, (2001), 200-213.

[16] S. V. Djordjević, I. H. Jeon and E. Ko, Weyl's theorem through local spectral theory, Glasgow Math. J. 44 (2002), 323-327.

[17] B. P. Duggal and S. V. Djordjević, Weyl's theorems and continuity of spectra in the class of p-hyponormal operators, Studia Math. 143 (2000), 23-32.

[18] —, - Generalized Weyl's theorems for a class of operators satisfying a norm condition, Math. Proc. Roy. Irish Acad. Sect. A 104 (2004), 75-82.

[19] N. Dunford, Spectral theory I. Resolution of the identity, Pacific J. Math. 2 (1952), 559-614.

[20] J. K. Finch, The single valued extension property on a Banach space, ibid. 58 (1975), $61-69$.

[21] T. Furuta, Invitation to Linear Operators, Taylor and Francis, London, 2001.

[22] Y. M. Han and A.-H. Kim, A note on *-paranormal operators, Integral Equations Operator Theory 49 (2004), 435-444.

[23] Y. M. Han and W. Y. Lee, Weyl's theorem holds for algebraically hyponormal operators, Proc. Amer. Math. Soc. 128 (2000), 2291-2296.

[24] H. Heuser, Functional Analysis, Marcel Dekker, New York, 1982.

[25] T. Kato, Perturbation theory for nullity, deficiency and other quantities of linear operators, J. Anal. Math. 6 (1958), 261-322.

[26] K. B. Laursen and M. M. Neumann, Introduction to Local Spectral Theory, Clarendon Press, Oxford, 2000.

[27] S. H. Lee and W. Y. Lee, A spectral mapping theorem for the Weyl spectrum, Glasgow Math. J. 38 (1996), 61-64.

[28] C. Lin, Y. Ruan and Z. Yan, p-hyponormal operators are subscalar, Proc. Amer. Math. Soc. 131 (2003), 2753-2759.

[29] M. Mbekhta, Sur la théorie spectrale locale et limite des nilpotents, ibid. 110 (1990), 621-631.

[30] M. Oudghiri, Weyl's and Browder's theorem for operators satisfying the SVEP, Studia Math. 163 (2004), 85-101.

[31] M. Putinar, Quasi-similarity of tuples with Bishop's property $(\beta)$, Integral Equations Operator Theory 15 (1992), 1047-1052.

[32] V. Rakočević, On the essential approximate point spectrum II, Mat. Vesnik 36 (1984), 89-97.

[33] -, Approximate point spectrum and commuting compact perturbations, Glasgow Math. J. 28 (1986), 193-198.

[34] P. Vrbová, On local spectral properties of operators in Banach spaces, Czechoslovak Math. J. 23 (98) (1973), 483-492.

Dipartimento di Metodi e Modelli Matematici

Università di Palermo

Viale delle Scienze

I-90128 Palermo, Italy

E-mail:paiena@unipa.it

Received January 20, 2004

Revised version February 1, 2005 